Classification

Physics Abstracts

$0.676-0.692-4.220$

\title{
APPLICATION DE LA TECHNIQUE DU JET D'HÉLIUM A L'IDENTIFICATION EN LIGNE DE NOUVEAUX NOYAUX PAR LA DÉTECTION DE LEURS RAYONNEMENTS GAMMA
}

\author{
C. DEPRUN, H. GAUVIN, B. LAGARDE et LE BEYEC \\ Laboratoire de Chimie Nucléaire, \\ Institut de Physique Nucléaire, \\ B. P. $n^{0} 1$, 91-Orsay, France
}

(Reçu le 18 février 1974, révisé le 28 mai 1974)

\begin{abstract}
Résumé. - La méthode d'entraînement des produits de réactions nucléaires par un jet d'hélium a été adaptée à la collection et à la détection de noyaux émetteurs $\gamma$. Cette méthode a été appliquée en utilisant un faisceau d'ions ${ }^{12} \mathrm{C}$ délivré par le Cyclotron à Ions Lourds d'Orsay. Les réactions ${ }^{103} \mathrm{Rh}\left({ }^{12} \mathrm{C}, x \mathrm{n}\right){ }^{115-x} \mathrm{Sb}$ ont été étudiées entre 50 et $90 \mathrm{MeV}$. Les isotopes d'antimoine de masse 113, 112,111 et 110 ont été identifiés par leurs rayonnements $\gamma$. Les développements possibles de la méthode sont examinés.

Abstract. - The transport method for nuclear reaction products by a helium jet has been modified for use in collecting and detecting $\gamma$ ray-emitters. This method has been applied using a ${ }^{12} \mathrm{C}$ ion beam delivered by the Orsay Heavy Ion Cyclotron. The reactions ${ }^{103} \mathrm{Rh}\left({ }^{12} \mathrm{C}, x \mathrm{n}\right){ }^{115-x} \mathrm{Sb}$ have been studied between 50 and $90 \mathrm{MeV}$. Antimony isotopes with masses 113-112-111 and 110 have been identified by their $\gamma$-rays. Possible improvements of this method are examined.
\end{abstract}

1. Introduction. - La technique du jet d'hélium a été essentiellement utilisée jusqu'à présent, soit pour l'étude de la production de nouveaux isotopes émetteurs alpha, soit pour l'étude de mécanismes de réactions nucléaires conduisant à des noyaux résiduels émetteurs alpha.

Cette méthode est maintenant utilisée pour la collection de noyaux émetteurs $\gamma$. La détection de rayonnements gamma émis par des noyaux, dont les périodes peuvent être comprises entre quelques milli-secondes et quelques secondes, offre des possibilités de recherches à la fois nouvelles et intéressantes; elles peuvent se classer en 3 rubriques : production et identification de nouveaux noyaux, études spectroscopiques avec mesure de coïncidence $\gamma-\gamma, \beta-\gamma, \alpha-\gamma$, étude de mécanismes de réactions nucléaires.

Des essais de détection de rayonnements gamma par cette méthode ont été faits récemment par $\mathrm{R}$. MacFarlane et al. [1]. Nous présentons ici les premiers résultats expérimentaux obtenus en utilisant un faisceau d'ions ${ }^{12} \mathrm{C}$ accélérés par le cyclotron à ions lourds d'Orsay, et les développements possibles de la méthode. Les réactions ${ }^{103} \mathrm{Rh}\left({ }^{12} \mathrm{C}, x \mathrm{n}\right){ }^{115-x} \mathrm{Sb}$ ont permis de produire et d'identifier, par leurs rayonnements gamma, les isotopes d'antimoine de masses 113, 112, 111 et 110 . Ces isotopes déficients en neutrons produits et mis en évidence récemment aux cours de réactions ( $\mathrm{p}, x \mathrm{n}$ ) avaient fait l'objet d'études spectroscopiques [2,
$3,4]$. Nos expériences ont confirmé les résultats précédents et ont surtout permis de tester la méthode pour les prochaines investigations de nouveaux noyaux.

2. Dispositif expérimental. - 2.1 PRINCIPE DE LA MÉTHODE. - Le système jet d'hélium, en fonctionnement à Orsay, a été décrit par ailleurs $[5,6]$. Le principe de la méthode peut être rappelé brièvement. Après réactions nucléaires induites par des ions lourds sur une cible, les noyaux produits sont éjectés de la cible et thermalisés dans de l'hélium maintenu à une pression de 1,5 à $2 \mathrm{~kg} / \mathrm{cm}^{2}$. Ces noyaux sont ensuite aspirés avec l'hélium à travers un tube capillaire de diamètre intérieur de l'ordre de $0,8 \mathrm{~mm}$ vers une enceinte où sont situés les détecteurs de rayonnements. Une pompe à grand débit maintient dans cette enceinte un vide de 3 à $5 \times 10^{-2}$ torr. Face à l'orifice de sortie du capillaire, et à faible distance, est placée une surface collectrice sur laquelle les noyaux entraînés viennent se fixer. Le dépôt a un diamètre d'environ $2 \mathrm{~mm}$.

2.2 RENDEMENT DE COLLECTION. - Le rendement de collection représente le rapport entre le nombre de noyaux collectés et le nombre de noyaux produits par réaction nucléaire. Il peut dépasser $50 \%$ mais dépend toutefois de plusieurs facteurs. Il a été montré $[7,8]$ que le transport par l'hélium des noyaux radio-actifs, pouvait être accru de façon impor- 
tante par l'adjonction d'impuretés à ce gaz. Les noyaux produits se lient à ces gros agrégats moléculaires, ainsi formés, de masses élevées (jusqu'à $10^{8}$ uma) avant d'être transportés par l'hélium. Des études récentes $(*)$ [9] montrent que ces agrégats peuvent d'abord être produits puis introduits dans la chambre à réaction par l'hélium.

Divers systèmes ont été essayés par d'autres auteurs, dont le plus perfectionné est un générateur d'aérosol (La Mer aerosol generator) [9] grâce auquel les impuretés introduites peuvent être choisies en fonction de leur réactivité particulière avec les éléments produits par réactions nucléaires.

Au cours d'essais, on a trouvé, pour notre part, que l'adjonction à l'hélium de trace de vapeur d'eau permettait d'accroître d'un facteur 2 le rendement de collection. Pour la mesure du rendement on a utilisé la réaction ${ }^{209} \mathrm{Bi}(\mathrm{C}, 4 \mathrm{n}){ }^{217} \mathrm{Ac} \stackrel{\alpha}{\rightarrow}{ }^{213} \mathrm{Fr}$ dont la section efficace absolue est de $30 \mathrm{mb}$ [10] pour l'énergie de bombardement $84 \mathrm{MeV}$. Une valeur de $60 \%$ a été trouvée pour le rendement global de collection.

2.3 DÉTECTION DES RAYONNEMENTS GAMMA. - Le dispositif expérimental utilisé pour la détection des rayonnements gamma des noyaux produits a subi peu de modification par rapport à celui utilisé pour la détection de la radioactivité $\alpha$. La figure 1 en présente

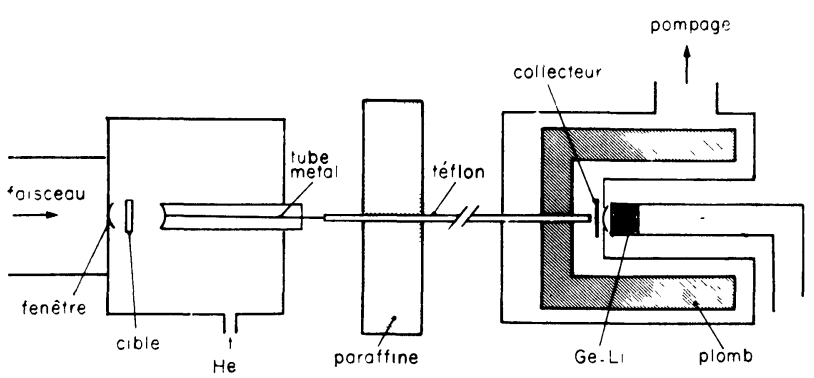

FIG. 1. - Schéma de principe du dispositif expérimental à jet d'hélium utilisé avec un détecteur $\mathrm{Ge}(\mathrm{Li})$.

un schéma d'ensemble. Dans les expériences que nous avons réalisées, le tube capillaire était constitué d'un tube métallique en acier inoxydable de longueur $30 \mathrm{~cm}$ et de diamètre intérieur $0,6 \mathrm{~mm}$, à la suite duquel on avait mis un tube de Téflon de diamètre intérieur $1,2 \mathrm{~mm}$ et de longueur $1,5 \mathrm{~m}$. Le débit de gaz dans ces conditions était de l'ordre de $50 \mathrm{cc} / \mathrm{s}$.

Les noyaux transportés par l'hélium sont déposés sur un collecteur d'Al situé à $5 \mathrm{~mm}$ d'une jonction $\mathrm{Ge}(\mathrm{Li})$ de $40 \mathrm{cc}$ de volume et de résolution globale $2,6 \mathrm{keV}$ à $1,33 \mathrm{MeV}$. Cette jonction a été réalisée au Laboratoire par L. Stab et ses collaborateurs.

Entre le détecteur et le collecteur, une fenêtre d'Al d'épaisseur $50 \mu$ sépare le vide de la chambre et l'air dans lequel est situé le détecteur.

(*) Wicsehahn, W. J., d'Auria, J. M., Dautet, H. and Pate, B. D., Communication Personnelle.

Afin de réduire le bruit de fond gamma, très important malgré la protection de plomb réalisée autour du détecteur, nous avons utilisé un système de pulsation du faisceau [6] et fait nos mesures entre les impulsions.

Dans ces conditions, nous avons bombardé une cible de ${ }^{103} \mathrm{Rh}$ par un faisceau d'ions carbone aux énergies de $89,84,76,67,60,54$ et $50 \mathrm{MeV}$. L'énergie maximale des ions délivrés par la machine est de $94 \mathrm{MeV}$ et nous avons réduit l'énergie jusqu'à ces valeurs en interposant sur le faisceau des feuilles d'Al d'épaisseur calculées d'après les tables de ralentissement de Northcliffe [11]. Enfin, pour chaque énergie de bombardement, un nouveau collecteur était mis en place devant le détecteur $\mathrm{Ge}(\mathrm{Li})$.

3. Résultats expérimentaux. - La figure 2 présente le spectre gamma obtenu à l'énergie de $67 \mathrm{MeV}$. Pour chacun des rayonnements observés, nous avons tracé, en fonction de l'énergie incidente, la variation de l'intensité liée au taux de production de l'isotope considéré, et ceci en tenant compte du facteur d'efficacité du détecteur. Les fonctions d'excitation ${ }^{103} \mathrm{Rh}(\mathrm{C}, x \mathrm{n})$ pour $x=2,3,4,5$ qui ont été déduites sont présentées sur la figure 3 . Les positions des seuils et des maxima de ces fonctions d'excitation permettent alors d'attribuer sans ambiguïté, les rayonnements observés aux différents isotopes produits.

De plus, aux énergies des ions ${ }^{12} \mathrm{C}$, correspondant aux maxima des fonctions d'excitation, les périodes radioactives ont été mesurées par la technique du multispectre en irradiations successives. Le procédé est celui décrit dans la référence [6].

La liste des rayonnements gamma, leurs pourcentages relatifs et les assignations en masse, sont présentés dans les tableaux I, II, III. Dans ces tableaux, figurent

$$
\begin{aligned}
& T_{1 / 2} \mathrm{~S} \\
& E_{\gamma}(\mathrm{keV}) \\
& 670 \\
& 895 \\
& 991 \\
& 1258
\end{aligned}
$$$$
T_{1 / 2} \mathrm{~S}
$$$$
E_{\gamma}(\mathrm{keV})
$$

\section{TABlEAU I ${ }^{112} \mathrm{Sb}$}

$$
\begin{array}{ccc}
\text { Réf. [3] } & \text { Réf. [2] } & \text { Présent travail } \\
-53,5 \pm 0,5 & 56 \pm 1 & 54 \pm 0,5 \\
& \text { intensités relatives } \\
3,1 \pm 1 & \multicolumn{3}{c}{3,4 \pm 0,7} \\
2,9 \pm 0,7 & & 3,1 \pm 0,4 \\
15 \pm 1 & 12,1 \pm 1,3 & 16,4 \pm 2 \\
100 & 100 & 100
\end{array}
$$

TABLEAU II

${ }^{111} \mathrm{Sb}$

$$
\begin{aligned}
& \text { Réf. [3] Présent travail } \\
& 74, \overline{1} \pm 1,4 \quad 75 \overline{\text { 土 }} 1 \\
& \text { intensités relatives } \\
& 7 \pm 2 \quad 6,7 \pm 1,5 \\
& 100 \quad 100 \\
& 32 \pm 2 \quad 69 \pm 12 \\
& 0,8 \pm 0,3 \quad 7,3 \pm 1,5 \\
& 10 \pm 2 \\
& 4 \pm 0,5 \quad 8,2 \pm 1,8
\end{aligned}
$$




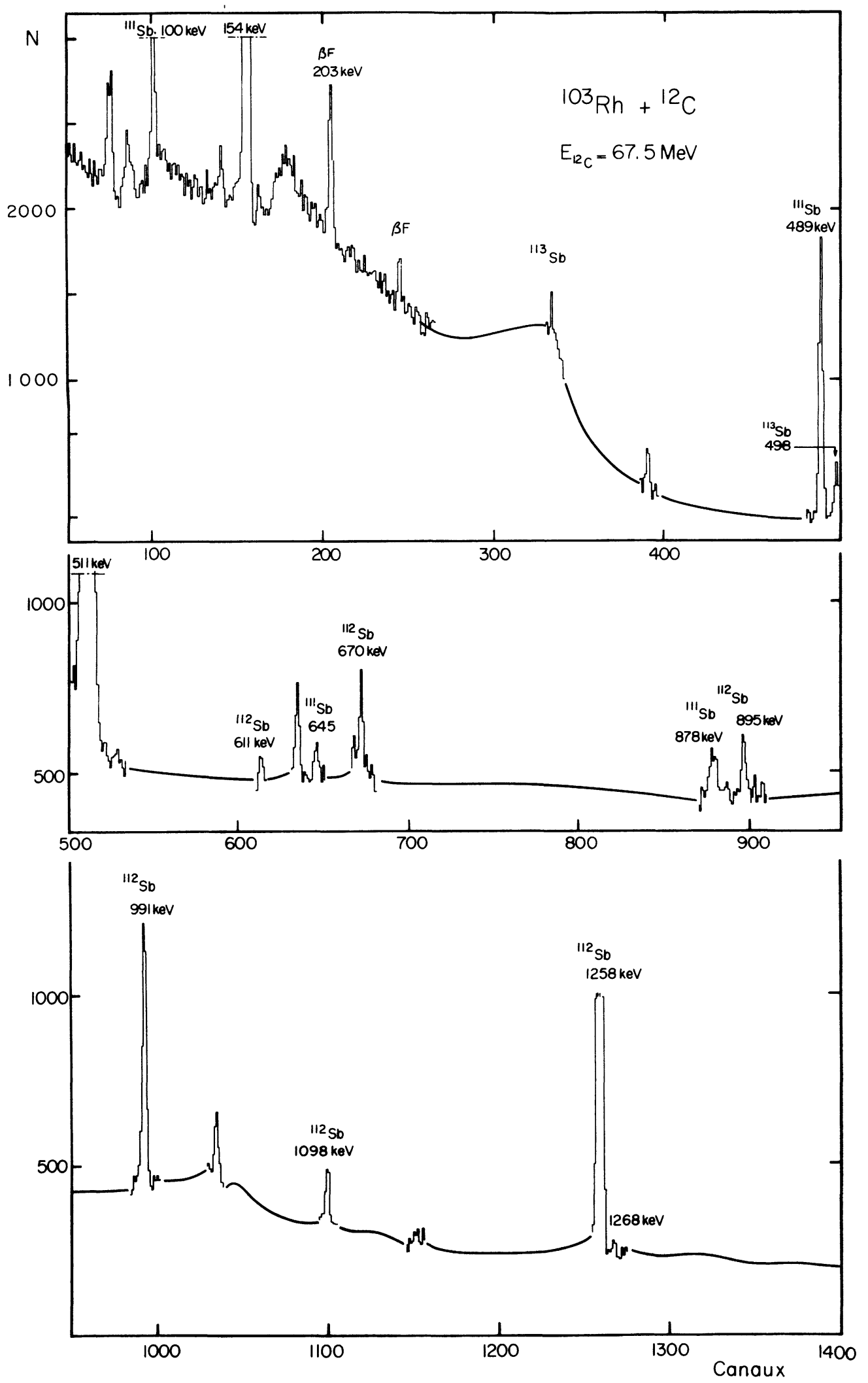

FIG. 2. - Réaction ${ }^{103} \mathrm{Rh}+{ }^{12} \mathrm{C}$. Spectre gamma enregistré à $67 \mathrm{MeV}$. Les isotopes produits, identifiés par leurs rayonnements $\gamma$ sont indiqués. Dans un souci de clarté, les parties du spectre ne comportant pas de pics $\gamma$ ont été représentées par un tracé continu. 


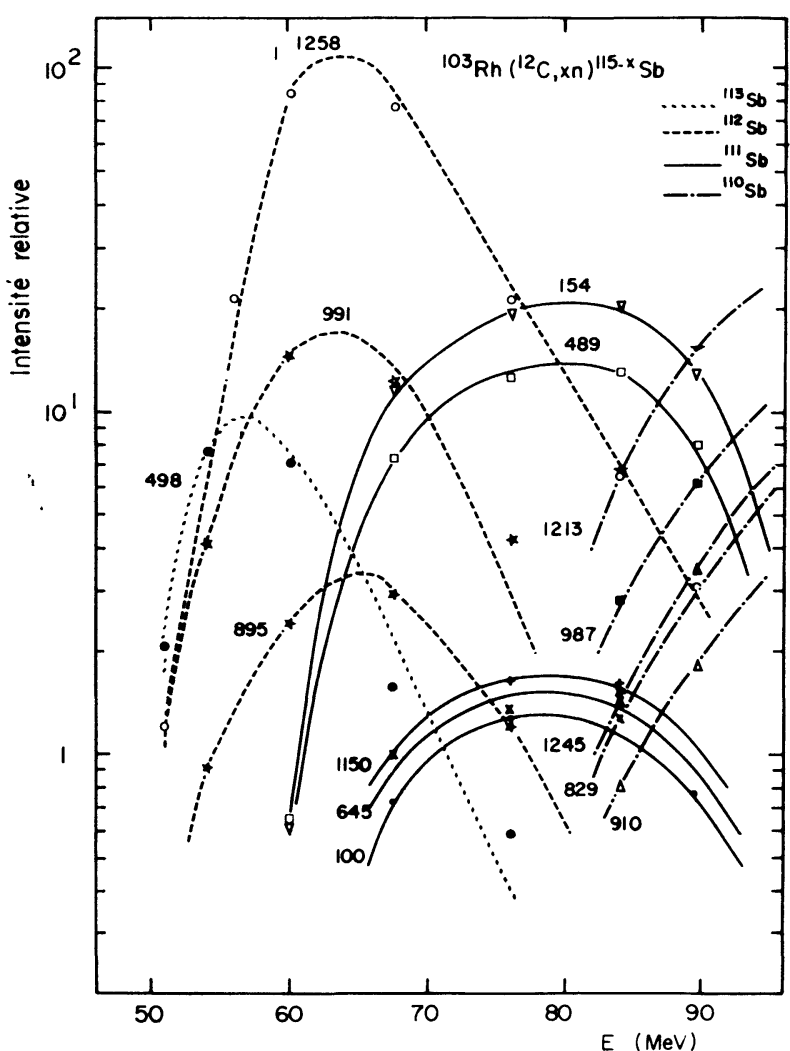

Fig. 3. - Fonctions d'excitation des rayonnements $\gamma$ observés. Identification des isotopes $113,112,111,110$ de $\mathrm{Sb}$ produits par ${ }^{103} \mathrm{Rh}\left({ }^{12} \mathrm{C}, x \mathrm{n}\right)$.

\section{TABLEAU III ${ }^{110} \mathrm{Sb}$}

\begin{tabular}{rccc} 
& Réf. [3] & Réf. [4] & Présent travail \\
$T_{1 / 2} \mathrm{~s}$ & $23,0 \pm 0,4$ & $21,3 \pm 1,5$ & $23 \pm \pm 1$ \\
$E_{\gamma}(\mathrm{keV})$ & & \multicolumn{2}{c}{ intensités relatives } \\
440 & pas observé & $19 \pm 0,5$ & pas observé \\
721 & pas observé & $15,4 \pm 0,9$ & pas observé \\
829 & $12 \pm 2$ & $15,1 \pm 1,2$ & $20 \pm 3,4$ \\
910 & $6,5 \pm 1$ & $14,7 \pm 1,3$ & $11,8 \pm 2,3$ \\
987 & $38 \pm 4$ & $44,1 \pm 1,8$ & $43 \pm 7$ \\
1213 & 100 & 100 & 100 \\
1245 & $14 \pm 2$ & $17,1 \pm 1$ & $22,3 \pm 4,3$
\end{tabular}

également les résultats des récents travaux. L'accord est en général satisfaisant, en particulier pour ${ }^{112} \mathrm{Sb}$. Quelques différences, parfois importantes, apparaissent pour certaines valeurs des intensités relatives dans les cas de ${ }^{111} \mathrm{Sb}$ et ${ }^{110} \mathrm{Sb}$, et restent inexpliquées, pour l'instant. L'origine de ces différences fait actuellement l'objet d'un travail distinct. Pour ${ }^{111} \mathrm{Sb}$, le rayonnement $\gamma$ de $1032 \mathrm{keV}$ (visible sur le spectre de la Fig. 2) ne nous est pas apparu avec une période de $75 \mathrm{~s}$. Aussi n'est-il pas indiqué dans le tableau II.

4. Discussion. - 4.1 Performances. - Ces résultats sont intéressants car ils montrent que la méthode mise en œuvre permet d'identifier des isotopes déficients en neutrons en mesurant les rayonnements gamma. Des améliorations sont à apporter notamment en ce qui concerne les limites inférieures de détection; elles seront obtenues par la diminution du bruit de fond. Pour cela, la solution consiste d'abord à éloigner le détecteur $\mathrm{Ge}(\mathrm{Li})$ du lieu de production des noyaux, en portant à 10 ou $20 \mathrm{~m}$ la longueur du capillaire. Il n'y aura pas d'atténuation du rendement de collection, si l'on conserve le débit de gaz en augmentant le diamètre intérieur du capillaire. De nombreuses mesures de débit ont d'ailleurs été réalisées par C. Deprun [12] qui a établi un réseau d'abaques permettant de choisir en fonction du débit désiré, la longueur et le diamètre intérieur du capillaire.

Il sera néanmoins nécessaire d'assurer un blindage efficace du ou des détecteurs $\mathrm{Ge}(\mathrm{Li})$.

On peut estimer actuellement que pour une cible d'épaisseur égale au parcours des reculs, des durées d'irradiation de l'ordre de $10 \mathrm{~min}$, une intensité de faisceau de $10^{11}$ particules par seconde, des périodes autour de $30 \mathrm{~s}$, notre limite inférieure de section efficace détectable par des rayonnements gamma d'énergie comprise entre 0,1 et $1,5 \mathrm{MeV}$ est de $20 \mathrm{mb}$ (les intensités absolues gamma étant prises égales à $10 \%$ ).

Ces valeurs peuvent servir de base à d'autres estimations. Par exemple un temps d'accumulation 10 fois plus grand permettra d'accéder toujours dans ces mêmes conditions, à des valeurs de $20 \mathrm{mb}$ pour des intensités absolues de $1 \%$. La réduction du bruit de fond permettra d'atteindre des valeurs beaucoup plus faibles de l'ordre de 3 à $5 \mathrm{mb}$ pour une intensité de $1 \%$. Ceci donne une idée des bonnes performances que l'on peut espérer atteindre dans la détection des rayonnements gamma.

4.2 Autres applications. - La mesure de rayonnements $X$ émis par les nucléides produits et collectés peut constituer un développement intéressant de cette technique. En effet, au cours de bombardements de cibles par des ions projectiles lourds comme ${ }^{16} \mathrm{O},{ }^{40} \mathrm{Ar}$, ${ }^{40} \mathrm{Ca}$ ou ${ }^{84} \mathrm{Kr}$ (faisceaux d'ions lourds accélérés par l'accélérateur Alice à Orsay) des réactions nucléaires complexes (noyau composé, transferts, fission) ont lieu conduisant à la production d'éléments de numéro atomique pouvant atteindre celui du noyau de fusion avec des sections efficaces plus ou moins importantes [13, 14]. L'identification de ces éléments dont les isotopes ont souvent des périodes radioactives courtes pourrait être faite dans certains cas grâce à la collection par jet d'Hélium suivie de mesures de rayonnements $X$.

De plus, en dehors de l'identification de nouveaux isotopes déficients en neutrons, il peut être envisagé des études purement spectroscopiques. En particulier, des mesures de coïncidences $\beta-\gamma, \gamma-\gamma$ et $\alpha-\gamma$ permettraient de préciser les schémas de niveaux des isotopes produits. Pour de telles expériences, il y aurait lieu d'envisager quelques modifications simples du système actuel. En particulier, il est possible de déposer l'activité sur une bande très fine (bande magnétique, par exemple) entraînée par un dérouleur. Le système permettrait, d'une part, d'une irradiation à l'autre, le changement 
automatique de collecteur pour éviter la mesure des produits de filiation et, d'autre part, le positionnement des noyaux radioactifs entre deux détecteurs fonctionnant en coïncidence.

En ce qui concerne l'étude de mécanismes de réactions nucléaires, les travaux effectués jusqu'à maintenant $[5,15,16,17]$ étaient liés à la détection des noyaux résiduels émetteurs alpha, ce qui limitait la méthode à certaines zones de noyaux produits. La mesure de rayonnement gamma offre par contre un domaine beaucoup plus vaste. Il restera cependant à choisir des rayonnements gamma sélectifs, pour l'identification des noyaux produits.

Enfin, cette méthode n'est pas restrictive aux seules réactions par ions lourds. On a déjà montré que l'utilisation de faisceau de particules légères telles que ${ }^{3} \mathrm{He}$ permettait d'obtenir d'excellents résultats dans un temps très court. Des noyaux de bismuth très déficients en neutrons $(190<A<197)$ ont été produits par réaction ${ }^{203} \mathrm{Tl}\left({ }^{3} \mathrm{He}, x \mathrm{n}\right)$ et leurs caractéristiques de désintégrations initialement mal connues ont été déterminées avec précision [18, 19]. De même l'étude de réactions ${ }^{206} \mathrm{~Pb}\left({ }^{3} \mathrm{He}, x \mathrm{n}\right){ }^{209-x} \mathrm{Po}$ pour $8<x<14$ a été menée au synchrocyclotron d'Orsay en utilisant la méthode d'entraînement par jet d'hélium [20]. Le rendement global de collection était de $10 \%$ seulement, mais là encore des améliorations peuvent être apportées. Il apparaît donc tout à fait envisageable d'utiliser des faisceaux d'ions légers pour produire des noyaux dont on mesurerait la désexcitation gamma. Il sera cependant absolument indispensable dans ce cas de situer le détecteur loin du faisceau et de la cible.

\section{Bibliographie}

[1] Bowman, W. W., Sugihara, T. T. and MacFarlane, R. D., Nucl. Instrum. Methods 103 (1972) 61.

[2] Kazumasa, M. and Carmen, G., J. Phys. Soc. Japan 33 (1972) 1509.

[3] Singh, M., Sunier, J. W., Devries, R. M. and Thompson, G. E., Nucl. Phys. A 193 (1972) 449.

[4] Kazumasa, M., Hiromichi, N. and Carmen, G., J. Phys. Soc. Japan 33 (1972) 1505.

[5] Le Beyec, Y., Lefort, M., SARda, M., Nucl. Phys. A 192 (1972) 404

[6] Livet, J., Thèse de $3^{\mathrm{e}}$ cycle, IPN Orsay (1973).

[7] Jungclas, H., MacFarlane, R. D. and Farès, Y., Radiochim. Acta 16 (1971) 141.

[8] Jungclas, H., MacFarlane, R. D. and Farès, Y., Phys. Rev. Lett. 27 (1971) 556.

[9] Wilhelm, H. G., Jungclas, H., Wollnik, H., Snider, D. F., BRandt, R., RöBig, G., International Report Philipps Universität, Marburg, Germany (1973).

[10] Sarda-Gugliermina, M., Thèse de $3^{\mathrm{e}}$ cycle, IPN Orsay (1972).
[11] NorthCliffe, C. and Schilling, R. F., Nuclear Data Tables A 7 (1970) 233.

[12] Deprun, C., travail non publié.

[13] Bimbot, R., Gardès, D., Hahn, R. L., de Moras, Y. and RIVET, M. F., IPNO-RC-74.01.

[14] Gauvin, H., Le Beyec, Y., Porile, N. T., Nucl. Phys. A 223 (1974) 103.

[15] Gauvin, H., Lefort, M., Le Beyec, Y. and Deprun, C., Phys. Rev. Lett. 28 (1972) 697.

[16] Gauvin, H., Le Beyec, Y. and Porile, N. T., Proceed. Eur. Conf. on Nucl. Phys., Aix-en-Provence, II (1972) 92.

[17] Gauvin, H., Hahn, R. L., Lagarde, B., Le Beyec, Y., Lefort, M , Nucl. Phys. A 220 (1974) 84.

[18] Gauvin, H., Le Beyec, Y., Lefort, M., Porile, N. T., Phys. Rev. Lett. 29 (1972) 958.

[19] Le Beyec, Y., Lefort, M., Livet, J., Porile, N. T., SiIvola, A., Phys. Rev. C 9 (1974) 1091.

[20] ANDRÉ, C., Thèse $3^{\mathrm{e}}$ cycle, IPN Orsay (1973). 